\title{
Anakinra erhält EU-Zulassung für die Behandlung des Still-Syndroms
}

Die Europäische Kommission hat die Zulassung von Kineret ${ }^{\circledast}$ (Anakinra) um die Behandlung des Still-Syndroms erweitert. Die Zulassungserweiterung umfasst sowohl die systemische juvenile idiopathische Arthritis (SJIA) als auch den Morbus Still des Erwachsenen (AOSD, adult-onset Still's disease).

Das Still-Syndrom ist eine seltene, systemische, autoinflammatorische Multiorgan-Krankheit, an der in der EU ca. 25.000 Kinder und Erwachsene leiden [1]. In Ergänzung der bisherigen lautet die neue Indikation: Kineret $^{\circledast}$ ist indiziert bei Erwachsenen, Jugendlichen, Kindern und Kleinkindern ab acht Monaten mit einem Körpergewicht von $10 \mathrm{~kg}$ und mehr zur Behandlung des Still-Syndroms einschließlich der systemischen juvenilen idiopathischen Arthritis (SJIA) und des Morbus Still des Erwachsenen (AOSD, adult-onset Still's disease) mit aktiven systemischen Merkmalen von moderater bis hoher Krankheitsaktivität oder bei Patienten mit fortbestehender Krankheitsaktivität nach Behandlung mit nicht-steroidalen Antirheumatika (NSARs) oder Glukokortikoiden. Kineret ${ }^{\circledast}$ kann als Monotherapie gegeben werden oder in Kombination mit anderen anti-entzündlichen Medikamenten oder sogenannten DMARDs (disease-modifying antirheumatic drugs).

„Wir sind sehr erfreut über die Zulassungserweiterung von Kineret für das Still-Syndrom in der EU. Sie beinhaltet die Option, Kineret als Alternative zu Steroiden als First-line-Therapie einzusetzen. Dadurch schließt sich eine Lücke in der medizinischen Versorgung der Patienten mit Still-Syndrom", sagt Milan Zdravkovic, Chief Medical Officer und Leiter von Forschung und Entwicklung bei Swedish Orphan Biovitrum GmbH (Sobi).

Auch Prof. Dr. Johannes-Peter Quelle: PresseausOrphan Biovitrum $\mathrm{GmbH}$ gendrheumatologie (DZKJR) in Garmisch-Partenkirchen begrüßt die Zulassungserweiterung von Anakinra: „Die Zulassung von Kineret ${ }^{\circledR}$ für die Behandlung der systemischen juvenilen idiopathischen Arthritis und des adulten Morbus Still ist sehr begrüßenswert. Kineret wird seit mehr als zehn Jahren sehr erfolgreich zur Behandlung der SJIA eingesetzt und hat sich als ein wirksames und sicheres Medikament erwiesen. Die gute Dosierbarkeit ermöglicht es, auch sehr kleine Patienten zu behandeln, bzw. sich in der Dosierung an den individuellen Erfordernissen des Patienten und seines Erkrankungsverlaufes zu orientieren. Daher ist die Zulassung ab dem achten Lebensmonat wichtig und gerechtfertigt. Die Zulassung zum Einsatz auch ohne vorherige Glukokortikoidtherapie entspricht den Erfordernissen einer modernen steroidsparenden Therapie der SJIA."

Die Zulassungserweiterung basiert sowohl auf Studiendaten als auch auf wissenschaftlicher Literatur und Meta-Analysen der publizierten Daten. Insgesamt basiert die Bewertung auf Daten von mehr als 400 Patienten mit Still-Syndrom. Sie zeigt die Wirksamkeit von Anakinra sowohl bei Kindern als auch bei Erwachsenen mit StillSyndrom. Die Mehrheit der Patienten erreichte eine Remission und eine Verbesserung der Krankheitssymptome [2].

Die aus den klinischen Studien und der publizierten Literatur über das Still-Syndrom gewonnenen Daten zur Anwendungssicherheit von Kineret belegen ebenso wie die seit 2002 ge- sammelten post-Marketing Daten über die Anwendung von Kineret sowohl beim Still-Syndrom als auch anderen Indikationen ein gutes Sicherheitsprofil. Die häufigsten Nebenwirkungen sind Reaktionen an der Einstichstelle, Kopfschmerzen und eine Erhöhung des Gesamtcholesterins [3].

„Diese Zulassungserweiterung ist ein wichtiger Meilenstein für uns. Kineret ist seit langem bekannt als ein wirksames und effektives Medikament zur Behandlung von rheumatoider Arthritis und dem Cryopyrin-assoziierten periodischen Syndrom (CAPS). Mit dieser neuen Indikation erhalten Patienten mit Still-Syndrom Zugang zu einer alternativen Therapie mit einem guten Sicherheitsprofil. Wir konzentrieren uns jetzt auf einen schnellen Launch in Europa, mit dem wir in Deutschland, Großbritannien, den Niederlanden und in Nordeuropa beginnen," sagt Norbert Oppitz, Senior Vice President Specialty Care bei Sobi.

\section{Literatur}

1. Interne Schätzung von Sobi

2. New treatment option for rare inflammatory disease, EMA Press release, 23 February 2018.

3. European prescribing information, EMA website

rheuma plus $2018 \cdot 17: 78$ https://doi.org/10.1007/s12688018-0186-y

(c) Springer-Verlag GmbH Austria, ein Teil von Springer Nature 2018 